\title{
XMAP215 is a long thin molecule that does not increase microtubule stiffness
}

\author{
Lynne Cassimeris ${ }^{1,2, \ddagger}$, David Gard ${ }^{3}$, P. T. Tran ${ }^{4, \star}$ and Harold P. Erickson ${ }^{2}$ \\ ${ }_{1}^{1}$ Department of Biological Sciences, Lehigh University, Bethlehem, PA, USA \\ ${ }^{2}$ Cell Biology Department, Duke University School of Medicine, Durham, NC, USA \\ ${ }^{3}$ Biology Department, University of Utah, Salt Lake City, UT, USA \\ ${ }^{4}$ Biology Department, University of North Carolina, Chapel Hill, NC, USA \\ *Present address: Department of Microbiology, Columbia University, New York, NY, USA \\ ₹Author for correspondence (e-mail: Ic07@lehigh.edu) \\ Accepted 17 May 2001 \\ Journal of Cell Science 114, 3025-3033 (2001) @ The Company of Biologists Ltd
}

\section{SUMMARY}

XMAP215 is a microtubule associated protein that speeds microtubule plus end growth by seven- to tenfold and protects these ends from destabilization by the Kin I kinesin, XKCM1. To understand the mechanisms responsible for these activities, it is necessary to know the structure of XMAP215. By unidirectional shadowing and electron microscopy, XMAP215 appeared as an elongate molecule of $60 \pm 18 \mathrm{~nm}$, suggesting that XMAP215 could span up to seven to eight tubulin dimers along a protofilament. Most XMAP215 molecules were straight but a subset were bent suggesting that XMAP215 is flexible. Antibodies to the C terminus labeled one end of XMAP215 with no evidence for XMAP215 dimerization. Incubation of XMAP215 and tubulin at $4^{\circ} \mathrm{C}$ resulted in assembly of curved protofilaments, which appeared to be incomplete tubulin rings. Measurements from rotary shadowed samples showed that tubulin/XMAP215 partial rings had an average width of $8.8 \pm 1.8 \mathrm{~nm}$ compared with $5.6 \pm 1.1 \mathrm{~nm}$ for rings assembled from tubulin dimers alone, suggesting that XMAP215 adds a width of approximately $3.2 \mathrm{~nm}$ to the curved tubulin protofilament. XMAP215 did not change the radius of curvature of these partial tubulin rings. Measurements of microtubule flexural rigidity by thermal fluctuations showed that XMAP215 did not change microtubule rigidity. Finally, sequence analysis shows that the N-terminal half of XMAP215 contains four repeats, each composed of multiple HEAT repeats.

Key words: Microtubules, MAPs, Electron microscopy, Flexural rigidity

\section{INTRODUCTION}

Microtubule polymers are dynamic and continually turn over by processes of dynamic instability and/or treadmilling. Dynamic instability is the major pathway of microtubule turnover in cells and describes a two-state behavior of microtubules ends; these ends can exist in either a growth or a shortening state. Abrupt transitions between states are termed catastrophe (the switch from growth to shortening) and rescue (the switch from shortening to growth). Treadmilling, the preferential gain and loss of subunits from opposite microtubule ends, has also been observed in cells (WatermanStorer and Salmon, 1997; Rodionov and Borisy, 1997).

These dynamic turnover mechanisms are probably regulated by microtubule associated proteins (MAPs), as turnover in vivo can be faster than that observed with purified tubulin in vitro (reviewed by Desai and Mitchison, 1997). One such regulator is XMAP215, a protein initially purified from Xenopus egg extracts, based on its ability to stimulate microtubule plus-end growth (Gard and Kirschner, 1987). Subsequent studies have demonstrated that XMAP215 speeds microtubule plus-end assembly seven- to tenfold, increases shortening rate threefold, and does not change catastrophe frequency (Vasquez et al., 1994). By contrast, XMAP215 had negligible effects on minusend assembly (Vasquez et al., 1994). Therefore, it is likely that one function of XMAP215 is to speed microtubule plus-end growth, yet allow rapid microtubule turnover in cells. This has been demonstrated in egg extracts where partial depletion of XMAP215 results in approximately twofold slower growth rate in interphase extracts (Tournebize et al., 2000), while 90\% depletion nearly eliminates microtubule assembly (Tournebize et al., 2000; Popov et al., 2001). XMAP215 also protects microtubule plus ends from the catastrophe-promoting activity of the Kin I kinesin, XKCM1 (Tournebize et al., 2000). XKCM1 and related kinesins are thought to disrupt microtubule ends by peeling away protofilaments and then releasing tubulin dimers by an ATP-dependent mechanism (Desai et al., 1999). How XMAP215 is able to both speed plusend assembly and protect microtubule ends from XKCM1 is not understood.

XMAP215 probably functions during spindle assembly, as depletion of this MAP abolishes spindle assembly in Xenopus egg extracts (Tournebize et al., 2000; Wilde and Zheng, 1999). The importance of XMAP215 function during mitosis is also supported by studies of related members of this MAP family: genetic mutations in Drosophila mini-spindles protein (Msps; Cullen et al., 1999), C. elegans Zyg-9 (Matthews et al., 1998), S. cerevisiae Stu2p (Wang and Huffacker, 1997) and S. pombe p93 dis1 (Nabeshima et al., 1995; Nabeshima et al., 1998) resulted in defective spindle assembly, short microtubules or poor viability. Finally, depletion of the human homolog, TOGp, from HeLa cell extracts prevented mitotic aster 
assembly (Dionne et al., 2000). XMAP215 and the related MAP family members probably function in mitosis by stimulating microtubule plus-end assembly and counteracting the destabilizing activity of Kin I kinesins (Tournebize et al., 2000), but additionally XMAP215 may serve as a scaffold to localize cyclin $\mathrm{B} / \mathrm{CDK} 1$ to microtubules during mitosis, as has been observed for TOGp (Charrasse et al., 2000).

Recent sequence analysis suggests that the XMAP215 family members contain a large number of HEAT repeats (Neuwald and Hirano, 2000). While the primary amino acid sequence is not well conserved, each HEAT repeat forms a common structural motif consisting of two $\alpha$ helices that associate together in an antiparallel orientation (reviewed by Kobe et al., 1999). The function of HEAT repeats is not known, but may contribute significantly to the structure of XMAP215.

To develop models of how XMAP215 functions as a regulator of microtubule assembly dynamics or as a scaffold, it is necessary to understand its structure. In the results presented here, we have used electron microscopy to examine the structures of XMAP215 and the partial tubulin rings assembled with this MAP. Additionally, we measured the flexural rigidity of microtubules assembled with XMAP215 to determine whether this MAP stiffens the microtubule lattice. Finally, we use the positions of identified HEAT repeats to align repeated sequences within the N-terminal half of XMAP215.

\section{MATERIALS AND METHODS}

\section{Protein purification}

XMAP215 was isolated from Xenopus eggs, as described previously (Gard and Kirschner, 1987) with the modifications described in Vasquez et al. (Vasquez et al., 1994). XMAP215 in BRB80 (80 mM Pipes, $1 \mathrm{mM} \mathrm{MgCl}, 1 \mathrm{mM}$ EGTA supplemented with $1 \mathrm{mM}$ DTT, 0.1 mM PMSF, $0.1 \mu \mathrm{g} / \mathrm{ml}$ phenanthroline, $0.1 \mathrm{mM}$ benzamidine $\mathrm{HCl}$ and $0.1 \mu \mathrm{g} / \mathrm{ml}$ pepstatin) was maintained at $4^{\circ} \mathrm{C}$ and used within 3-4 days of the final purification step. The two XMAP215 preparations used here ranged from 50-100 $\mu \mathrm{g} / \mathrm{ml}$ concentration, based on comparative staining of XMAP215 and BSA on Coomassie Blue stained SDS gels. Becker and Gard (Becker and Gard, 2000) have identified two isoforms of XMAP215 that differ by alternative splicing. As the XMAP215 used here is isolated from eggs, all considerations of protein sequence use the maternal isoform because this is the isoform present in the egg.

Tubulin was purified from porcine brains as described previously (Vasquez et al., 1994). No MAPs were detected in this preparation after silver staining of over-loaded SDS-PAGE mini-gels (50 $\mu \mathrm{g} / \mathrm{lane}$; not shown).

\section{Glycerol gradient centrifugation}

Approximately $25 \mu \mathrm{g}$ of purified XMAP215 was applied to a $4.5 \mathrm{ml}$ linear gradient of 15 to $40 \%$ glycerol in $0.2 \mathrm{M}$ ammonium bicarbonate. Standards consisting of catalase (11.3 S), albumin (4.6 S) and ovalbumin (3.5 S) were loaded on a separate gradient. Samples were spun at $4^{\circ} \mathrm{C}$ for 16 hours at $130,000 \mathrm{~g}$ in an SW 55 rotor, fractionated and examined by Coomassie Blue staining of 10\% SDS-PAGE gels. The sedimentation coefficient for XMAP215 was calculated by comparison with the standards by assuming linear separation across the gradient. The maximum sedimentation value was calculated (Ohashi and Erickson, 1997) using 228,000 as the molecular weight of XMAP215 (Tournebize et al., 2000; Becker and Gard, 2000).

\section{Tubulin/XMAP215 partial rings}

Tubulin $(4 \mu \mathrm{M})$ with or without XMAP215 $(0.4 \mu \mathrm{M})$ was incubated in $0.1 \times \mathrm{BRB} 80,1 \mathrm{mM}$ GTP and $15 \%$ glycerol for 1 hour on ice before spraying onto mica and shadowing (described below). Additional tubulin samples $(5 \mu \mathrm{M})$ lacking XMAP215 were incubated in $0.1 \times$ BRB80, $1 \mathrm{mM}$ GTP and $30 \%$ glycerol for several hours on ice before spraying onto mica.

\section{C-terminal antibody to TOGp/XMAP215}

A C-terminal fragment of TOGp (bp 5421-5961) was cloned into pGEX2T-L (pGEX2T (Amersham) modified to include additional restriction sites; a generous gift from $\mathrm{C}$. Larroque), transformed into Escherichia coli strain BL21 and expressed as a GST-fusion protein after induction by IPTG. The fusion protein was purified on a glutathione-S-transferase column according to the manufacturer's instructions. This GST-TOGp(amino acids 1807-1987) was used to immunize rabbits (Pocono Rabbit Farms) and generated antiserum that recognized a single band at $215 \mathrm{kDa}$ in HeLa lysates (data not shown). The antiserum was fractionated by ammonium sulfate precipitation (Harlowe and Lane, 1988) and then further purified on a $6 \times$ His-TOGp (amino acids 1807-1987) affinity column. The $6 \times$ Histagged TOGp (amino acids 1807-1987) was generated by cloning TOGp (bp 5421-5961) into the pQE30 vector (Qiagen) and expression of the fusion protein in E. coli strain M15. 6×His-TOGp (amino acids 1807-1987) was purified on a nickel column (Qiagen) and coupled to CNBr-activated Sepharose (Pharmacia), according to the protocol provided by the manufacturer. Antibodies were bound to the column in PBS and eluted with $0.1 \mathrm{M}$ glycine, $\mathrm{pH} 2.5$ and each $1 \mathrm{ml}$ fraction was neutralized with $200 \mu$ Tris- $\mathrm{HCl}, \mathrm{pH} 8.5$ (Harlowe and Lane, 1988). To demonstrate the specificity of the antibody for XMAP215, purified XMAP215 and a crude Xenopus egg extract were separated on $3-12.5 \%$ SDS PAGE gels, transferred to Immobilon membrane (Millipore) and probed with the purifed antibodies; antibody binding was detected by enhanced chemilumenescence (Amersham Pharmacia Biotech, Arlington Heights, IL).

\section{Shadowing and electron microscopy}

The peak XMAP215 fraction from the glycerol gradient was diluted twofold with $15 \%$ glycerol in ammonium bicarbonate and sprayed onto $\sim 1 \mathrm{~cm}$ square pieces of freshly cleaved mica. The mica was then dried under vacuum in a Balzers BAE 120 operated at room temperature. Specimens were rotary or uni-directional shadowed by evaporation of platinum onto the mica at $\sim 6^{\circ}$ and then coated with carbon at $90^{\circ}$. The platinum/carbon replicas were removed from the mica by flotation on deionized $\mathrm{H}_{2} \mathrm{O}$ and mounted on 400 mesh EM grids.

Replicas were examined using a Philips 301 electron microscope operated at $80 \mathrm{kV}$. Micrographs were taken at a magnification of $\sim 47,000-50,000 \times$. Magnification was calibrated using a negatively stained preparation of bovine tropomyosin paracrystals (Erickson et al., 1981).

\section{Image analysis}

EM negatives were scanned at 600 dpi resolution using an Epson Perfection 1200 scanner in negative mode. Measurements were made from the digital images using NIH image software. A distance of 2 $\mathrm{nm}$ was subtracted from each length or width measurement to remove the contribution from the shell of metal. The radius of curvature for partial rings was measured using Canvas 6.0 by drawing and overlaying a circle on the partial ring. The radius of each circle was then measured using the radius tool in Canvas. These latter measurements were not corrected for the platinum/carbon coating, as the circle was drawn to bisect the center of the arc of each partial ring. Statistical analysis was performed using analysis of variance in Microsoft Excel.

\section{Measurement of flexural rigidity by thermal flucuations}

For an idealized beam (i.e. a linear rod of homogeneous material and no structural variation), the curvature along its axis gives information about the bending moment associated with the impact of external bending energy. Bending moment and bending energy are coupled by Young's 
elastic modulus (E) and the geometric moment of inertia (I) (Feynman et al., 1964). For an idealized beam, these two parameters can be separated. However, a microtubule, made of subunits of $\alpha \beta$-tubulin, is not an idealized beam, and its elasticity is often specified by the product $E I$, which is a measurement of a the stiffness or flexural rigidity of the microtubule. Several techniques have been used previously to measure the flexural rigidity of microtubules by correlating bending energy with positional deflections of the microtubule long axis. Bending has been induced by passive excitation through thermal energy (Gittes et al., 1994; Venier et al., 1994; Mickey and Howard, 1995), or by controlled forces applied by optical trapping or hydrodynamic flow (Venier et al., 1994; Kurachi et al., 1995; Felgner et al., 1996; Felgner et al., 1997).

In the present study, microtubule flexural rigidity was derived from measurements of thermal fluctuations at the free ends of clamped microtubules. This type of flexural rigidity analysis relies the equation (see Appendix for derivation):

$$
E I=\frac{k_{\mathrm{B}} T L^{3}}{3\left\langle d^{2}\right\rangle},
$$

which suggests that the flexural rigidity $E I$ of a microtubule and the mean square deflection $\left\langle d^{2}\right\rangle$ of an axis point at a distance $L$ from the clamped end are inversely proportional (Venier et al., 1994). This equation assumed that $\mathrm{L}$ is much smaller than the persistence length of the microtubule $(\sim 6000 \mu \mathrm{m}$, Gittes et al., 1994). The remaining parameters are the absolute temperature $(T)$ and the Boltzman constant $\left(k_{\mathrm{B}}\right)$.

Microtubules were assembled from axoneme fragments and imaged using video-enhanced DIC microscopy at video rates (30 frames/second; Vasquez et al., 1994). Tubulin was assembled at 10 $\mu \mathrm{M}$ with or without $0.2 \mu \mathrm{M}$ XMAP215. The effects of XMAP215 on dynamic instability have been previously published (Vasquez et al., 1994) and the video tapes were analyzed here for rigidity by measuring microtubule thermal vibration. Axonemes adhered to the glass surface and therefore acted as a clamped point of the microtubule. Microtubule plus ends of less than $10 \mu \mathrm{m}$ length and free to vibrate, owing to thermal fluctuation, were selected for analysis. S-VHS tape sequences were digitized and captured into a computer (Power Macintosh) equipped with an image frame grabber board (Scion LG-3 PCI) and running the NIH-Image software. The capture rate was 15 frames/second, which translates to 67 msecond intervals between successive frames. For each series of frames describing one microtubule, an arc line perpendicular to the microtubule long axis was drawn near the vibrating microtubule tip. For each advancing image frame, the cursor was overlaid on the image of the microtubule lattice intersecting the arc line, and the $x$ $y$ coordinates of the position of interest were recorded. The point $\left(x_{0}\right.$, $y_{0}$ ) represents the position of the microtubule at the axoneme attachment site. The 50 collected points $\left(\left(x_{1}, y_{1}\right), \ldots,\left(x_{50}, y_{50}\right)\right)$ represent the positions of the vibrating tip of the microtubule for each of the 50 successive frames. The 50 points represent a spread of the different positions of the microtubule tip to the left and right of a mean position ( $\left.x_{\text {mean }}, y_{\text {mean }}\right)$, where:

$$
\begin{aligned}
& x_{\text {mean }}=\frac{1}{50} \sum_{i}^{50} x_{\mathrm{i}} . \\
& y_{\text {mean }}=\frac{1}{50} \sum_{i}^{50} y_{\mathrm{i}} .
\end{aligned}
$$

The mean-squared deflection $\left\langle d^{2}\right\rangle$ is calculated as:

$$
\left\langle d^{2}\right\rangle=\frac{1}{50} \sum_{i}^{50} d^{2},
$$

where,

$$
d^{2}=\left(x_{i}-x_{\text {mean }}\right)^{2}+\left(y_{i}-y_{\text {mean }}\right)^{2}
$$

The length $\mathrm{L}$ of the microtubule is calculated as:

$$
\mathrm{L}=\sqrt{ }\left\{\left(x_{0}-x_{\text {mean }}\right)^{2}+\left(y_{0}-y_{\text {mean }}\right)^{2}\right\}
$$

\section{RESULTS}

\section{XMAP215 is a long, thin protein}

Previous sedimentation and chromatography experiments had suggested that XMAP215 is an elongated protein (Gard and Kirschner, 1987). We confirmed this result by sedimentation of XMAP215 on a $15-40 \%$ glycerol gradient. In two experiments, XMAP215 sedimented between catalase (11.3 S) and albumin $(4.6 \mathrm{~S})$ at approximately $7 \mathrm{~S}$ (data not shown). The ratio of Smax to $\mathrm{S}$ is a useful guide to estimate the shape of proteins, where Smax is the sedimentation coefficient of an unhydrated sphere of radius sufficient to contain the mass of protein, and $\mathrm{S}$ is the measured sedimentation coefficient. Smax/S is equal to the frictional ratio, f/fmin, as defined by Tanford (Tanford, 1961); for globular proteins Smax/S is in the range of 1.2-1.3, while for elongated proteins it is 1.7-2.0 or higher (Tanford, 1961). For the $228 \mathrm{kDa}$ XMAP215, Smax is 14 (see Materials and Methods), giving Smax/S of 2.0. This ratio is the same as that found for a segment of tenascin containing eight fibronectin III domains, which is $25 \mathrm{~nm}$ long by $2.5 \mathrm{~nm}$ in diameter (Schürmann et al., 2001). Therefore, we conclude that XMAP215 is a highly elongated protein.

The shape of XMAP215 was observed directly by electron microscopy after unidirectional shadowing and appeared as a long, thin molecule of uniform density along its length (Fig. 1A). A corresponding gel of a glycerol gradient fraction used for shadowing is shown in Fig. 1B. Based on electron microscopy, we found no evidence for globular domains within the protein. In general, most XMAP215 molecules had a straight conformation, but several molecules were bent, suggesting that XMAP215 had some flexibility. The average length of an XMAP215 molecule was $60.0 \pm 17.7 \mathrm{~nm}$ s.d. $(n=86)$ (Fig. 1C).

Gard and Kirschner (Gard and Kirschner, 1987) have previously concluded that XMAP215 is a monomer in solution, based on results from size-exclusion chromatography and sucrose gradient sedimentation. To confirm that XMAP215 is a monomer and ensure that the above length measurement reflects the length of a single protein, we incubated XMAP215 with an affinity purified antibody raised against the C-terminus of TOGp. This antibody also recognizes XMAP215 (Fig. 2A). After unidirectional shadowing, antibody labeling was detected as a knob at one end of the protein (Fig. 2B) and the size of the knob $(\sim 13.5 \mathrm{~nm})$ is consistent with the size of single antibody molecules observed by others (Wille et al., 1992). No evidence for XMAP215 dimerization was observed, consistent with previous hydrodynamic experiments (Gard and Kirschner, 1987) and it is therefore highly likely that the long rods shown in Fig. 1A represent the individual XMAP215 molecules.

Based on the molecular mass of XMAP215 (228,000; Tournebize et al., 2000, Becker and Gard, 2000) and the length $(60 \mathrm{~nm})$ of a single molecule, XMAP215 has a mass density of $\sim 3800 \mathrm{Da} / \mathrm{nm}$. This mass density is approx. twofold higher than that determined for other MAPs (see Table 1 for comparison). 


\section{XMAP215 binds partial tubulin rings}

To examine whether XMAP215 can bind tubulin dimers or oligomers, XMAP215 and tubulin were incubated on ice to prevent microtubule assembly. Samples were then rotary shadowed and observed by electron microscopy. Under these conditions, addition of XMAP215 resulted in assembly of partial tubulin rings (Fig. 3). These partial rings were not observed in the absence of XMAP215 (Fig. 3). To compare the XMAP215/tubulin partial rings with those assembled from tubulin alone, we found that a higher glycerol concentration (30\%) and a longer incubation time (several hours) resulted in partial ring assembly from pure tubulin (Fig. 3).

The partial rings assembled with XMAP215 appeared uniform in width and were wider than rings assembled from tubulin alone (Fig. 4A); XMAP215/tubulin partial rings had an average width of $8.8 \pm 1.8 \mathrm{~nm}$ (s.d.; $n=59$ ) compared with $5.6 \pm 1.1$ (s.d.; $n=64$ ) for the tubulin partial rings. These mean widths are statistically different $(P<$ 0.01 ) and suggest that XMAP215 adds a width of approximately $3.2 \mathrm{~nm}$ to the curved tubulin protofilament. The lengths of the rings (Fig. 4B) also differed significantly $(P<0.01)$; rings assembled from tubulin and XMAP215 measured $59.6 \pm 26.2 \mathrm{~nm}$ s.d. $(n=86)$ compared with $48.6 \pm 24.8 \mathrm{~nm}$ (s.d.; $n=133$ ) for tubulin alone.

The curvature of the partial rings was also measured by determining the radius of a circle overlapping the arc formed by the partial rings (Fig. 4D). The partial rings had an average radius of curvature of $26.9 \mathrm{~nm}$ for tubulin alone and $26.8 \mathrm{~nm}$ for samples containing XMAP215 (Fig. 4C). These measured values are close to those reported previously for a protofilament ring (Voter and Erickson, 1979; Mandelkow et al., 1991). Clearly, the presence of XMAP215 had no effect on the curvature of the partial rings.

\section{Microtubule bending stiffness is not altered by XMAP215}

To determine whether XMAP215 affected the stiffness of microtubules, we measured microtubule plus end tip deflections and used these displacements to calculate microtubule flexural rigidity (see Materials and Methods). Vibration measurements were made on microtubule plus ends elongating from axoneme fragments in the absence or presence of XMAP215. Plus-end assembly was stimulated eightfold using the maximium possible concentration of XMAP215. Under these conditions, plus-end microtubules assembled without XMAP215 had a flexural rigidity of $17.5 \pm 2.2 \quad\left(10^{-24} / \mathrm{Nm}^{2} ; n=27\right)$ compared with $18.5 \pm 2.0\left(10^{-24} / \mathrm{Nm}^{2} ; n=25\right)$ for microtubules assembled from tubulin alone. Thus, XMAP215 did not change microtubule stiffness.

\section{Four N-terminal repeats in XMAP215 are composed of HEAT repeats}

Previous alignments of the XMAP215 family protein sequences have recognized that the segment corresponding to XMAP215 264-543 is highly conserved in all family members (Graf et al., 2000; Popov et al., 2001). Cullen et al. (Cullen et
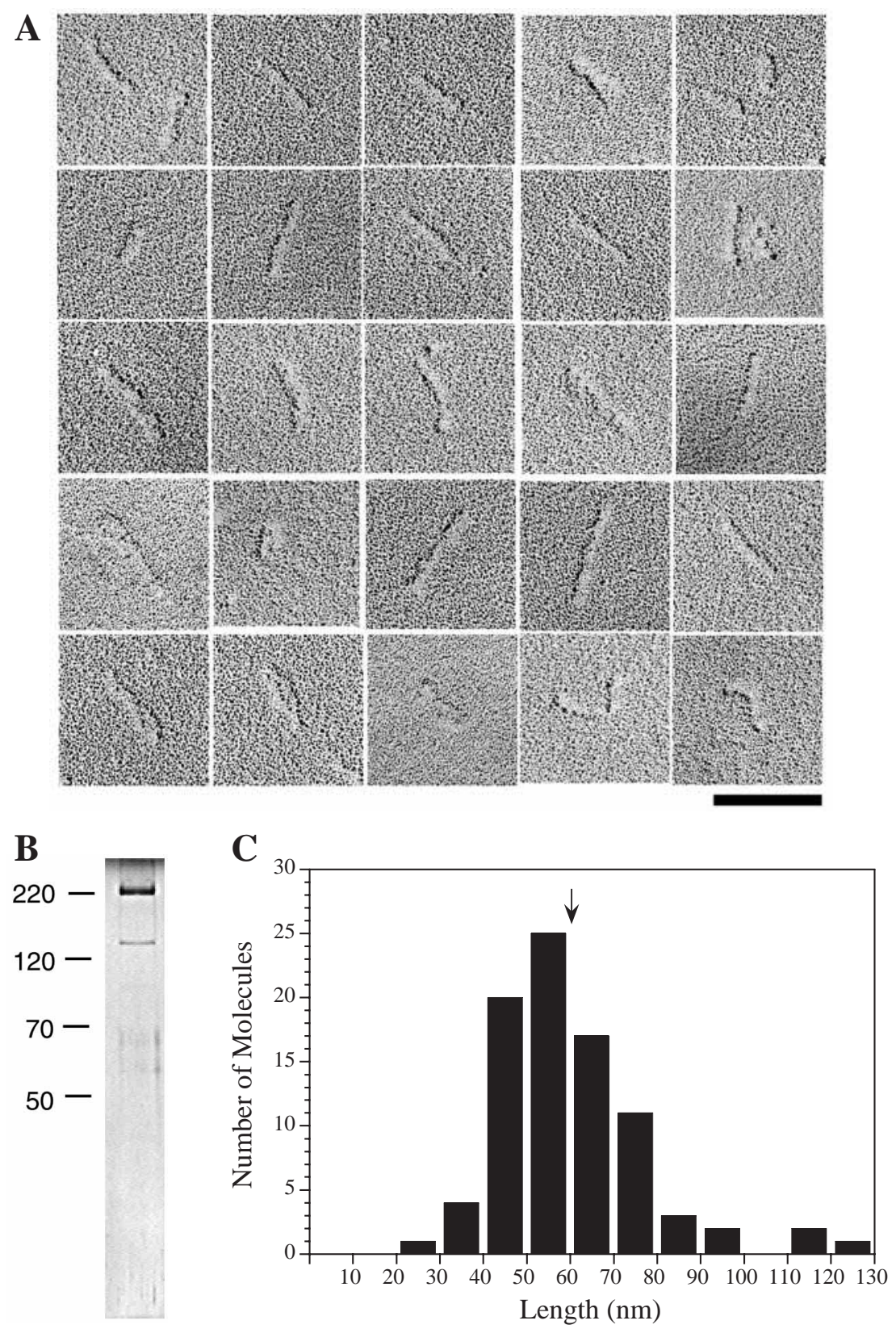

Fig. 1. XMAP215 is a long rod-shaped molecule. (A) Gallery of uni-directional shadowed images of XMAP215 selected from two different XMAP215 preparations. Samples were sprayed onto mica and shadowed with platinum/carbon as described in the Materials and Methods. Most molecules appeared relatively straight, but a significant number were bent (e.g. bottom row), suggesting that XMAP215 is a flexible molecule. Scale bar: $100 \mathrm{~nm}$. (B) Corresponding SDS-PAGE gel of the peak glycerol fraction used for shadowing. A small fraction of the sample was proteolyzed; this is commonly observed with XMAP215. The positions of $M_{\mathrm{r}}$ markers are shown. (C) Histogram of XMAP215 length distribution. The lengths of individual XMAP215 molecules were measured from digitized EM negatives of uni-directional shadowed XMAP215. The mean XMAP215 length of $60 \mathrm{~nm}$ is marked with an arrow. 
XMAP215 structure

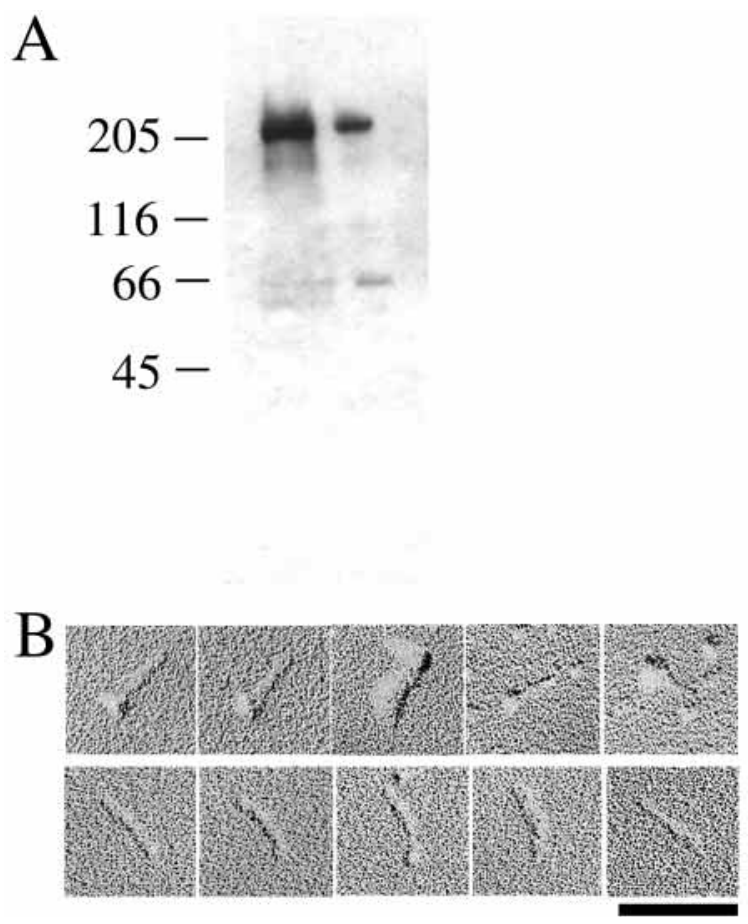

Fig. 2. XMAP215 is a monomer. (A) Immunoblot of purified XMAP215 and a Xenopus egg extract probed with an antibody raised against the $\mathrm{C}$ terminus of TOGp. The antibody recognizes purified XMAP215 and a $215 \mathrm{kDa}$ protein in Xenopus egg extracts. Positions of $M_{\mathrm{r}}$ markers are shown. (B) XMAP215 was incubated with (top row) or without (bottom row) an antibody to its $\mathrm{C}$ terminus before uni-directional shadowing. In the presence of antibody, the XMAP215 rod was decorated by a single globular structure that had the size of an antibody molecule, suggesting that XMAP215 is a monomer. Scale bar: $100 \mathrm{~nm}$.

al., 1999) noted further that there were actually four repeats of this subdomain in the $\mathrm{N}$-terminal region of TOGp and Drosophila Msps. They identified four separate motifs that made a common signature for the subdomain, but the overall sequence identity between the four subdomains is quite limited, making alignment difficult. Recent sequence analysis suggests that the XMAP215 family members contain a large
Table 1. Mass per unit length for XMAP215 and other MAPs

\begin{tabular}{lcrcl}
\hline Protein & $\begin{array}{c}\text { Mass } \\
(\mathrm{Da})\end{array}$ & $\begin{array}{c}\text { Length } \\
(\mathrm{nm})\end{array}$ & $\begin{array}{c}\text { Mass } \\
\text { density } \\
(\mathrm{Da} / \mathrm{nm})\end{array}$ & Refs \\
\hline XMAP215 & 228,373 & 60 & 3806 & Present study \\
MAP2 & 198,859 & 180 & 1105 & Voter and Erickson, 1982 \\
& & 97 & 2050 & Wille et al., 1992 \\
MAP1B & 270,453 & 186 & 1454 & Sato-Yoshitake et al., 1989 \\
CLIP-170 & 156,698 & 135 & 1160 & Scheel et al., 1999 \\
Tau & $36,737-45,822$ & 35 & $1469-1309$ & Schweers et al., 1994
\end{tabular}

Mass density calculations used the predicted molecular weights determined by sequence analysis; therefore some values listed above will differ from previously published calculations.

number of HEAT repeats (Neuwald and Hirano, 2000; see Discussion). We found that use of these poorly conserved HEAT repeat sequences provided a guide for alignment within the N terminus of XMAP215 and that this analysis removed most ambiguities. As shown in Fig. 5A, there are five HEAT repeats in each of the four $\mathrm{N}$ terminal subdomains (N1-N4), and possibly a sixth one following these not recognized by sequence. The arrangement of HEAT repeats and the subdomains is shown in Fig. 5B.

\section{DISCUSSION}

The results of our EM observations showed that XMAP215 is a thin, rod-shaped molecule of approximately $60 \mathrm{~nm}$ length (Fig. 1A,C). The sedimentation coefficient of $7 \mathrm{~S}$ measured in our study is close to the $5 \mathrm{~S}$ reported by Gard and Kirschner (Gard and Kirschner, 1987). Both values are consistent with the highly elongated shape seen by EM. The width of the XMAP215 molecule could not be accurately measured, as we used uni-directional shadowing to visualize the protein. However, we could estimate the width of XMAP215 from the difference in width between partial tubulin rings assembled in the presence and absence of XMAP215 (Figs 3, 4); these results suggest that XMAP215 is approximately $3.2 \mathrm{~nm}$ wide. The thickness of XMAP215 can also be estimated from its mass density of approximately $3800 \mathrm{Da} / \mathrm{nm}$. If XMAP215
Fig. 3. XMAP215 binds partial tubulin protofilament rings. Tubulin $(4 \mu \mathrm{M})$ was incubated with XMAP215 $(0.4 \mu \mathrm{M})$ in $0.1 \times \mathrm{BRB} 80$ and $15 \%$ glycerol for 1 hour on ice before rotary shadowing. Under these conditions, a fraction of the tubulin assembled into partial rings (top row). In the absence of XMAP215, rings were not detected and the observed molecules were approximately the size of single tubulin dimers (middle row). Tubulin could be assembled into partial rings without XMAP215 (bottom row) by increasing the glycerol concentration (30\%) and incubation time (several hours). Scale bar: $100 \mathrm{~nm}$.
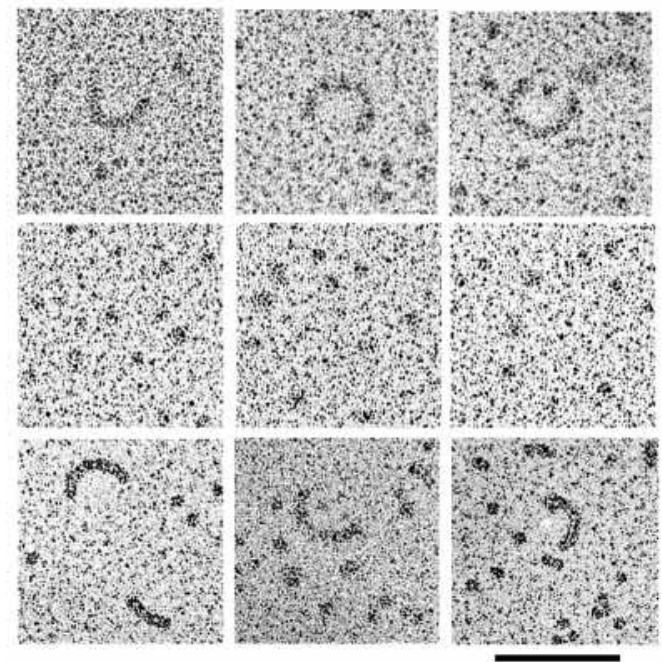

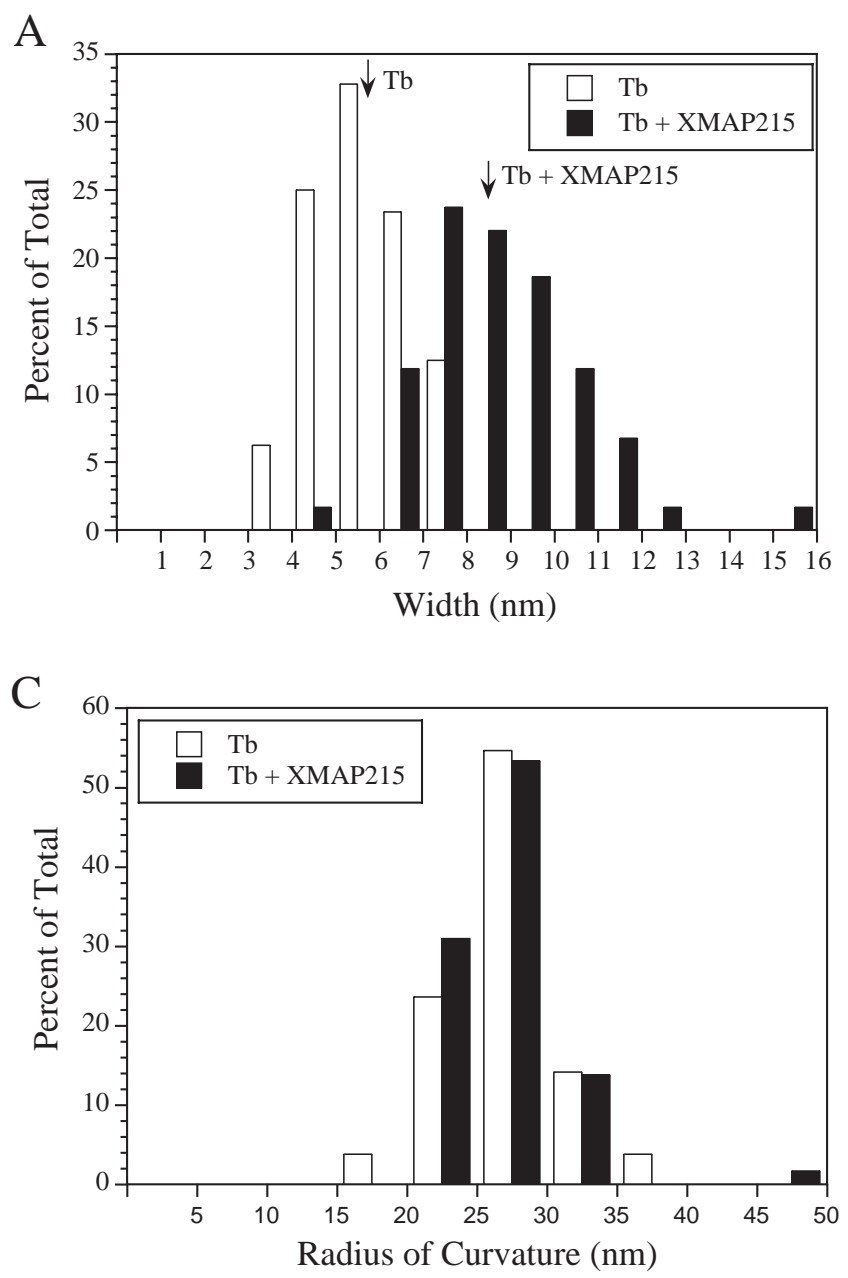

were a compact cylinder of protein with a density of 1.37 $\mathrm{g} / \mathrm{cc}$, it would have a diameter of $1.3 \mathrm{~nm}$. This would be the smallest possible diameter for a cylinder of this mass and length. If the molecule had a more irregular folding or domain structure, the diameter could easily be 2-3 times greater.

The binding of XMAP215 to partial tubulin rings suggests that XMAP215 binds along a single protofilament, since a tubulin ring consists of a curved protofilament (Voter and Erickson, 1979). This conclusion is consistent with results from experiments with TOGp, demonstrating binding to microtubules, zinc sheets and tubulin rings (Spittle et al., 2000). The $60 \mathrm{~nm}$ length of XMAP215 suggests that this MAP could span seven to eight dimers along a protofilament. It is remarkable that the length of the partial tubulin rings assembled with XMAP215 (59.6 nm) is identical to the average length of the MAP (60 nm), suggesting that tubulin dimers may have bound all along the length of a single XMAP215 molecule.

Our results are thus consistent with evidence that XMAP215/TOGp binds to microtubules with a large surface of the MAP bound to the microtubule lattice. Microtubule binding assays of TOGp and fragments demonstrated that one microtubule-binding domain was located within a region of approximately 600 amino acids near the $\mathrm{N}$ terminus and a second region in the $\mathrm{C}$-terminal half of the protein was able to

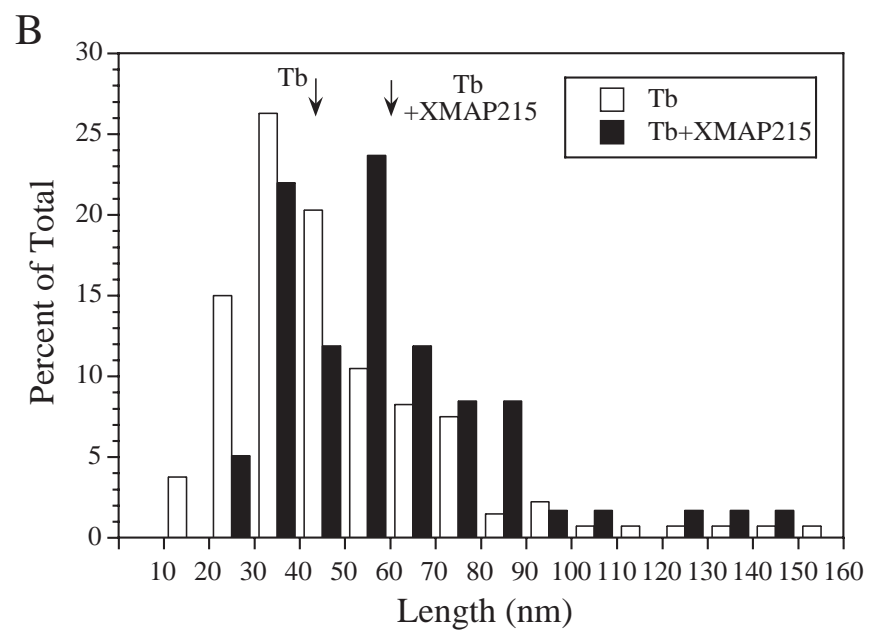

$\mathrm{D}$

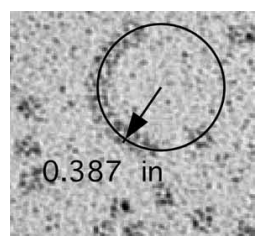

Fig. 4. XMAP215 increases the width (A) and length (B) of partial tubulin rings. By contrast, XMAP215 does not change the radius of curvature of the protofilament rings (C). (D) A partial ring and the circle overlay used to measure the radius. The radius is shown in inches on the micrograph and was then converted to nm. For A,B, the means are denoted by arrows. Histograms are presented as the percent of the total sample falling within each range to compensate for differences in sample size.

bind tubulin dimers or oligomers (Spittle et al., 2000). Expression of truncated segments of XMAP215 in vivo also suggests that the entire protein participates in microtubule binding (Popov et al., 2001). Taken together, these results suggest a widely distributed microtubule binding interface on XMAP215 and are consistent with recent insights into the structure of this protein, as discussed below.

\section{XMAP215 is composed of multiple HEAT repeats}

Recent iterative sequence analysis identified 20 HEAT repeats in TOGp (Neuwald and Hirano, 2000) and we find that these are also present in XMAP215 (Fig. 5A; data not shown). To compare our EM structure with the structures of well-defined HEAT repeat proteins, we used RASMOL (Sayle and MilnerWhite, 1995) to measure the spacings of HEAT repeats in PR65/A (PDB \# 1B3U), a subunit of PP2A that contains 15 tandem HEAT repeats (Groves et al., 1999). The spacing of 1.15 $\mathrm{nm}$ corresponds to a mass density of approximately 3750 $\mathrm{Da} / \mathrm{nm}$. This value compares well with the $3800 \mathrm{Da} / \mathrm{nm}$ estimated for XMAP215 (Table 1). Additionally, PR65/A has a depth of $2 \mathrm{~nm}$ and a width of $3.5 \mathrm{~nm}$ (Groves et al., 1999), similar to the $3.2 \mathrm{~nm}$ width we estimate for XMAP215, based on its binding to partial tubulin rings. Finally, HEAT repeat proteins are thought to be flexible (Kobe et al., 1999), which is consistent with the variable bending seen in our EM of XMAP215. 
A

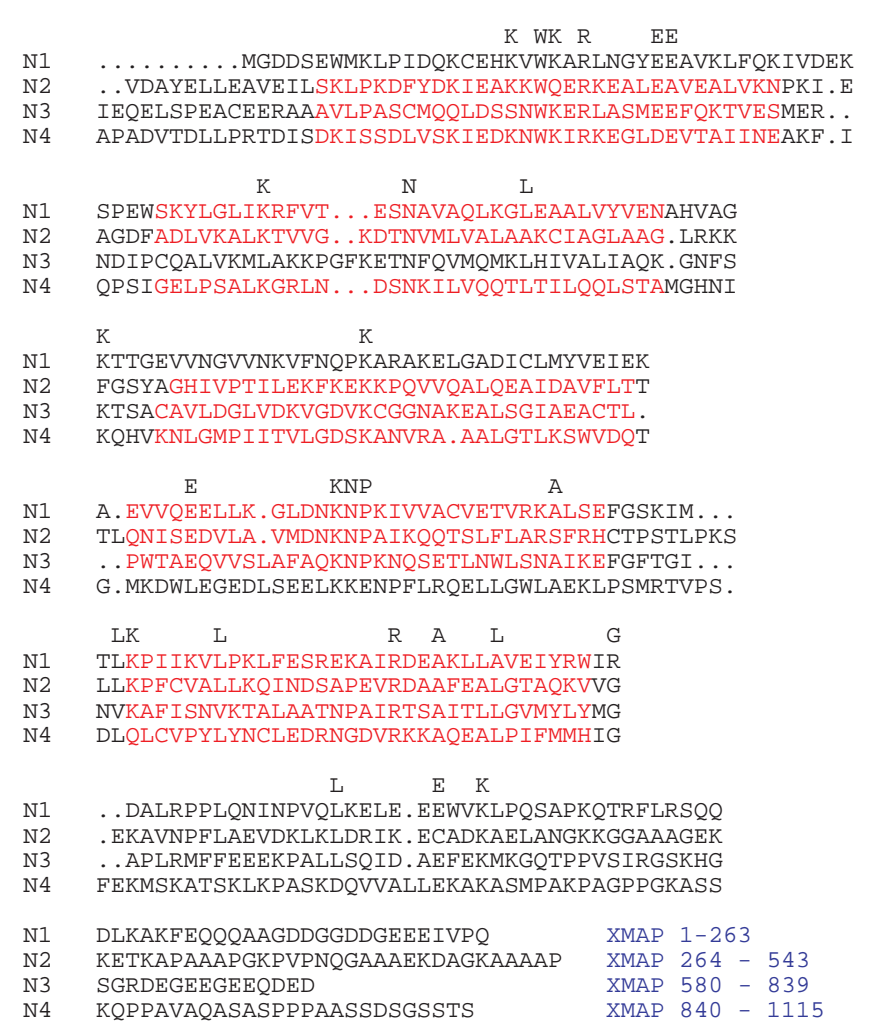

B

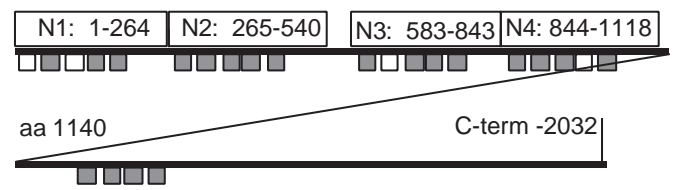

Fig. 5. (A) Alignment of the four subdomains within the N-terminal half of XMAP215, labeled N1-N4 and identified specifically on the last line. Amino acids conserved in three or four of the subdomains are indicated above the alignment. The HEAT repeats identified in TOGp by Neuwald and Hirano (Neuwald and Hirano, 2000) are shown in red (we transferred the repeats from TOGp to the closely related XMAP215 sequence). This alignment shows four segments where three subdomains have HEAT repeats, suggesting that the fourth subdomain also has one, not identified by the sequence analysis. A sixth HEAT repeat may occur in the final part of each subdomain, but this is not recognizable from the sequence. (B) A diagram of the TOGp sequence showing the location of HEAT repeats as dark boxes. The positions are nearly identical in

XMAP215. A 36 amino acid insert in XMAP215 (Becker and Gard, 2000 ) is located N-terminal to the last four identified repeats. The other XMAP215 insert is located C-terminal to the identified HEAT repeats. The dark boxes below indicate HEAT repeats identified by Neuwald and Hirano (Neuwald and Hirano, 2000), and the open boxes indicate additional repeats suggested by the alignment in A. The sub-domains N1-N4 are indicated above.

Based on the above considerations of sequence, mass density, shape and flexibility, and the fact that XMAP215 appears in the EM to have a uniform structure over its entire length, we propose that XMAP215 may be composed entirely of HEAT repeats. Additional HEAT repeats are likely to be of such divergent sequence that they have not yet been identified by the iterative search and Gibbs sampling alignment procedures used by Neuwald and Hirano (Neuwald and Hirano, 2000). Therefore, TOGp and XMAP215 could be composed of up to 45 HEAT repeats. HEAT repeat proteins typically act as scaffold proteins (Kobe et al., 1999) and a scaffold function for TOGp has been proposed recently (Charrasse et al., 2000). Finally, it is intriguing that another HEAT repeat protein, importin $\beta$ (Cingolani et al., 1999), has been identified as an intermediate in Ran-dependent microtubule assembly (Wiese et al., 2001; Nachury et al., 2001), as has XMAP215 (Wilde and Zheng, 1999), suggesting that these two HEAT repeat proteins may share binding partners.

\section{Microtubule stiffness}

Measurements of microtubule tip displacements were used to calculate microtubule flexural rigidity and demonstrated that XMAP215 did not alter microtubule stiffness. Thus, XMAP215 is able to speed plus-end assembly without altering microtubule stiffness. Several studies have measured microtubule flexural rigidity using several different methods; while the measured values differ, neuronal MAPs consistently stiffen microtubules (Kurachi et al., 1995; Mickey and Howard, 1995; Felgner et al., 1996; Felgner et al., 1997). The method used here also detected stiffening of microtubules when they bound neuronal MAPs (P. T. T. and E. D. Salmon, unpublished), suggesting that the lack of stiffening observed with XMAP215 is not due simply to the thermal vibration method used here. Thus, XMAP215 is the first MAP studied that does not stiffen microtubules and demonstrates that MAPs need not stiffen the microtubule lattice to stimulate assembly.

Several possible mechanisms could explain the lack of microtubule stiffening by XMAP215. First, XMAP215 may not be present at sufficient concentration to saturate the microtubule, although it was present in sufficient concentration to increase microtubule growth rate eightfold. Second, XMAP215 may be bound preferentially to microtubule ends where it would not affect microtubule stiffness. Preferential binding to microtubule ends has been observed with TOGp (Spittle et al., 2000). Third, XMAP215 may bind along the protofilament axis (discussed above); this has also been suggested for TOGp (Spittle et al., 2000).

\section{How does XMAP215 stimulate assembly and protect ends from depolymerization by XKCM1?}

Our results and those of Spittle et al. (Spittle et al., 2000) show that XMAP215/TOGp binds along the protofilament axis. In addition, XMAP215 does not appreciably stiffen the microtubule lattice under conditions that promote rapid plusend assembly. How, then, does XMAP215 stimulate plus end assembly? It seems unlikely that XMAP215 stimulates assembly through an interaction with the existing microtubule lattice. Rather, it is possible that XMAP215 enhances dimer addition to microtubule plus ends, as suggested previously (Gard and Kirschner, 1987; Spittle et al., 2000).

XMAP215 also counteracts the destabilizing activity of XKCM1. As XMAP215 does not function to stiffen the microtubule lattice and therefore does not increase longitudinal bond strength along a protofilament, XMAP215 cannot block $\mathrm{XKCM} 1$ action by simply increasing the energy necessary to remove a tubulin dimer from the microtubule end. XMAP215 
may compete for binding sites at microtubule ends and sterically hinder XKCM1 access to the microtubule. Alternatively, Popov et al. (Popov et al., 2001) have suggested that XKCM1 and XMAP215 may interact when not bound to the microtubule. In this case XMAP215 and microtubule ends would compete for binding to XKCM1.

In summary, XMAP215 is a thin protein that has a size and shape consistent with a structure based on multiple HEAT repeats. This structure provides an extended interface for interaction with the microtubule lattice or other binding partners. The proposed domain structure may provide the basis for making smaller expression proteins for biochemical and structural studies; this may lead to an understanding of how XMAP215 functions to speed assembly and protect ends from $\mathrm{XKCM} 1$.

\section{APPENDIX}

\section{Thermal fluctuation analysis theory}

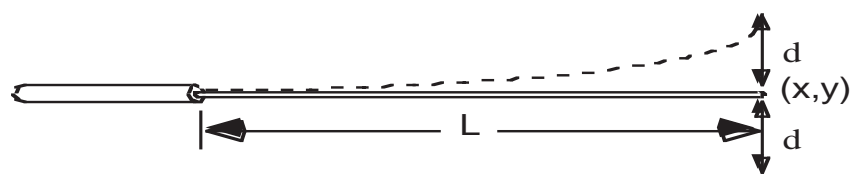

Fig. 6 shows a schematic drawing of a microtubule clamped at one end by attachment to the axoneme, and free at the other end to vibrate in solution due to thermal fluctuation. Let the microtubule profile be represented by the function $\mathrm{y}(\mathrm{x})$. The curvature (i.e. inverse of radius-of-curvature $R$ ) of $\mathrm{y}(\mathrm{x})$ is given by the equation:

$$
\frac{1}{R}=\frac{\frac{d^{2} y}{d x^{2}}}{\left[1+\left(\frac{d y}{d x}\right)^{2}\right]^{3 / 2}},
$$

If the curvature is large (i.e. the deflection $d$ is small) then we can assume $(d y / d x=0)$ and Eqn 1 reduces to:

$$
\frac{1}{R}=\frac{d^{2} y}{d x^{2}} \text {. }
$$

The bending moment $M$ (torque about the neutral axis of any cross section) of the microtubule at a given point from the origin is:

$$
M(x)=F[L-x]=\frac{E I}{R}=E I \frac{d^{2} y}{d x^{2}},
$$

where $F=$ perpendicular force vector at $x, L=$ total length of beam and $E I=$ flexural rigidity. The flexural rigidity $E I$ is the product of $E$, the Young's elastic modulus, and $I$, the moment area of inertia. Rearranging Eqns 2 and 3, we get:

$$
\frac{d^{2} y}{d x^{2}}=\frac{F[L-x]}{E I} .
$$

We can integrate Eqn. 4 twice to yield,

$$
\int \frac{d^{2} y}{d x^{2}}=\frac{F}{E I}\left[L x-\frac{x^{2}}{2}\right]=\frac{d y}{d x},
$$

and,

$$
\int \frac{d y}{d x}=\frac{F}{E I}\left[\frac{L x^{2}}{2}-\frac{x^{3}}{6}\right]=y(x) .
$$

At $x=L$, Eqn 6 reduces to,

$$
y(L)=\frac{F L^{3}}{3 E I} .
$$

The quantity $y(L)$ is the displacement, $\mathrm{d}$, of the tip of the microtubule. The condition at the tip of the clamped microtubule is also analogous to Hooke's law for a spring. For a spring, the force and potential energy associated with a deflection through a distance $\mathrm{y}$ are:

$$
F(y)=\kappa y
$$

$$
\operatorname{PE}(y)=\frac{1}{2} \kappa y^{2},
$$

where $\kappa=$ the spring constant, and $y=\mathrm{d}$. Rearranging Eqns 7 and 8 we arrive at:

$$
\kappa=\frac{3 E I}{L^{3}} .
$$

According to the equipartition theorem (Reif, 1965), the mean potential energy is:

$$
\langle P E\rangle=\frac{1}{2} k_{\mathrm{B}} T,
$$

where $k_{\mathrm{B}}=$ Boltzman constant and $T=$ absolute temperature. So, from Eqn. 9,

$$
\langle P E\rangle=\frac{1}{2} \kappa\left\langle y^{2}\right\rangle .
$$

Rearranging Eqns 11 and 12, we get:

$$
\frac{1}{2} \kappa\left\langle y^{2}\right\rangle=\frac{1}{2} k_{\mathrm{B}} T \text {. }
$$

Substituting Eqn 10 into 13, and rearranging, we get:

$$
\frac{3 E I}{L^{3}}=\frac{k_{\mathrm{B}} T}{\left\langle y^{2}\right\rangle},
$$

or,

$$
E I=\frac{k_{\mathrm{B}} T L^{3}}{3\left\langle y^{2}\right\rangle},
$$

where $\left\langle y^{2}\right\rangle=\left\langle\mathrm{d}^{2}\right\rangle$ is the mean square displacement of the microtubule tip.

L. C. is grateful to the members of the Erickson laboratory for a delightful sabbatical leave and the members of the Salmon laboratory for a productive weekend visit. L. C. thanks Gregor Schürmann and David Anderson for help with gradients and shadowing; Carmen Lucaveche for help with electron microscopy; Cindy Spittle and Michelle Kratzer for help with antibody production and purification; and Laura Romberg for helpful discussions. Supported by grants from NIH (L. C. and H. P. E.) and NSF (D. L. G.). 


\section{REFERENCES}

Becker, B. E. and Gard, D. L. (2000). Multiple isoforms of the high molecular weight microtubule associated protein XMAP215 are expressed during development in Xenopus. Cell Motil. Cytoskel. 47, 282-295.

Charrasse, S., Lorca, T., Doree, M. and Larroque, C. (2000). The Xenopus XMAP215 and its human homologue TOG proteins interact with cyclin B1 to target p34cdc2 to microtubules during mitosis. Exp. Cell Res. 254, 249-256.

Cingolani, G., Petosa, C., Weis, K. and Muller, C. W. (1999). Structure of importin- $\beta$ bound to the IBB domain of importin-alpha. Nature 399, 221-229.

Cullen, C. F., Deak, P., Glover, D. M. and Ohkura, H. (1999). mini spindles: a gene encoding a conserved microtubule-associated protein required for the integrity of the mitotic spindle in Drosophila. J. Cell Biol. 146; 1005-1018.

Desai, A. and Mitchison, T. J. (1997). Microtubule polymerization dynamics. Annu. Rev. Cell Biol. 13, 83-117.

Desai, A., Verma, S., Mitchison, T. J., Walczak, C. E. (1999). Kin I kinesins are microtubule-destabilizing enzymes. Cell 96, 69-78.

Dionne, M. A., Sanchez, A. and Compton, D. A. (2000). ch-TOGp is required for microtubule aster formation in a mammalian mitotic extract. $J$. Biol. Chem. 275, 12346-12352.

Erickson, H. P., Carrell, N., McDonagh, J. (1981). Fibronectin molecule visualized in electron microscopy: a long, thin, flexible strand. J. Cell Biol. 91, 673-678.

Felgner, H., Frank, R. and Schliwa, M. (1996). Flexural rigidity of microtubules measured with the use of optical tweezers. J. Cell Sci. 109 , 509-516.

Felgner, H., Frank, R., Biernat, J., Mandelkow, E. M., Mandelkow, E., Ludin, B., Matus. A., Schliwa, M. (1997). Domains of neuronal microtubule-associated proteins and flexural rigidity of microtubules. J. Cell Biol. 138, 1067-1075.

Feynman, R. P., Leighton, R. B. and Sands, M. (1964). The Feynman Lectures in Physics II. Reading MA: Addison-Wesley.

Gard, D. L. and M. W. Kirschner. (1987). A microtubule-associated protein from Xenopus eggs that specifically promotes assembly at the plus-end. $J$. Cell Biol. 105, 2203-2215.

Gittes, F., Mickey, B., Nettleton, J. and Howard, J. (1993). Flexural rigidity of microtubules and actin filaments measured from thermal fluctuations in shape. J. Cell Biol. 120, 923-934.

Graf, R., Daunderer, C. and Schliwa, M. (2000). Dictyostelium DdCP224 is a microtubule-associated protein and a permanent centrosomal resident involved in centrosome duplication. J. Cell Sci. 113; 1747-1758.

Groves, M. R., Hanlon, N., Turowski, P., Hemmings, B. A. and Barford, D. (1999). The structure of the protein phosphatase 2A PR65/A subunit reveals the conformation of its 15 tandemly repeated HEAT motifs. Cell. 96, 99-110.

Harlowe, E. and Lane, D. (1988). Antibodies. A Laboratory Manual. Cold Spring Harbor, NY: Cold Spring Harbor Laboratory Press.

Kobe, B., Gleichmann, T., Horne, J., Jennings, I. G., Scotney, P. D. and Teh, T. (1999). Turn up the HEAT. Stucture 7, R91-R97.

Kurachi, M., Hoshi, M., Tashiro, H. (1995). Buckling of a single microtubule by optical trapping forces: direct measurement of microtubule rigidity. Cell Motil. Cytoskel. 30, 221-228.

Mandelkow, E. M., Mandelkow, E. and Milligan, R. A. (1991). Microtubule dynamics and microtubule caps: A time-resolved cryo-electron microscopy study. J. Cell Biol. 114, 977-991.

Matthews, L. R., Carter, P., Thierry-Mieg, D. and Kemphues, K. (1998). ZYG-9, A Caenorhabditis elegans protein required for microtubule organization and function, is a component of meitotic and mitotic spindle poles. J. Cell Biol. 141, 1159-1168.

Mickey, B. and Howard, J. (1995). Rigidity of microtubules is increased by stabilizing agents. J. Cell Biol. 130, 909-917.

Nabeshima, K., Kurooka, H., Takeuchi, M., Kinoshita, K., Nakaseko, Y. and Yanagida, M. (1995). p93dis1, which is required for sister chromatid separation, is a novel microtubule and spindle pole body-associating protein phosphorylated at the cdc2 target sites. Genes Dev. 9, 1572-1585.

Nabeshima, K., Nakagawa, T., Straight, A. F., Murray, A., Chikashige, Y., Yamashita, Y. M., Hiraoka, Y. and Yanagida, M. (1998). Dynamics of centromeres during metaphase-anaphase transition in fission yeast: Dis1 is implicated in force balance in metaphase bipolar spindle. Mol. Biol. Cell 9, 3211-3225.

Nachury, M. V., Maresca, T. J., Salmon, W. C., Waterman-Storer, C. M.,
Heald, R. and Weis, K. (2001). Importin beta is a mitotic target of the small GTPase Ran in spindle assembly. Cell 104, 95-106.

Neuwald, A. F. and Hirano, T. (2000). HEAT repeats associated with condensins, cohesins, and other complexes involved in chromosome-related functions. Genome Res. 10, 1445-1452.

Ohashi T and Erickson, H. P. (1997). Two oligomeric forms of plasma ficolin have differential lectin activity. J. Biol. Chem. 272, 14220-14226.

Popov, A. V., Pozniakovsky, A., Arnal, I., Antony, C., Ashford, A. J., Kinoshita, K., Tournebize, R., Hyman, A. A. and Karsenti, E. (2001). XMAP215 regulates microtubule dynamics through two distinct domains. EMBO J. 20, 397-410.

Reif, F. (1965). Fundamentals of Statistical and Thermal Physics. New York: McGraw-Hill.

Rodionov, V. I. and Borisy, G. G. (1997). Microtubule treadmilling in vivo. Science 275, 215-218.

Sayle, R. A. and Milner-White, E. J. (1995). RASMOL: biomolecular graphics for all. Trends Biochem.Sci. 20, 374.

Sato-Yoshitake, R., Shiomura, Y., Miyasaka, H. and Hirokawa, N. (1989). Microtubule associated protein 1B: molecular structure, localization, and phosphorylation-dependent expression in developing neurons. Neuron $\mathbf{3}$, 229-238.

Scheel, J., Pierre, P., Rickard, J. E., Diamantopoulos, G. S., Valetti, c., van der Goot, F. G., Haner, M., Aebi, U. and Kreis, T. E. (1999). Purification and analysis of authentic CLIP-170 and recombinant fragments. J. Biol. Chem. 274, 25883-25891.

Schürmann, G., Haspel, J., Grumet, M. and Erickson, H. P. (2001). Cell adhesion molecule L1 in folded (horseshoe) and extended conformations. Mol. Biol. Cell 12, 1765-1773.

Schweers, O., Schonbrunn-Hanebeck, E., Marx, A. and Mandelkow, E. (1994). Structural studies of tau protein and Alzheimer paired helical filaments show no evidence for $\beta$ structure. J. Biol. Chem. 269, 2429024297.

Spittle, C., Charrasse, S., Larroque, C. and Cassimeris, L. (2000). The interaction of TOGp with microtubules and tubulin. J. Biol. Chem. 275, 20748-20753.

Tanford, C. (1961). In Physical Chemistry of Macromolecules, pp 357. New York: John Wiley.

Tournebize, R., Popov, A., Kinoshita, K., Ashford, A. J., Rybina, S., Pozniakovsky, A., Mayer, T. U. Walczak, C. E., Karsenti, E. and Hyman, A. A. (2000). Control of microtubule dynamics by the antagonistic activities of XMAP215 and XKCM1 in Xenopus egg extracts. Nat. Cell Biol. 2, 13 19.

Vasquez, R. J., Gard, D. L. and Cassimeris, L. (1994). XMAP from Xenopus eggs promote rapid plus end assembly of microtubules and rapid microtubule polymer turnover. J. Cell Biol. 127, 985-993.

Vasquez, R. J., Gard, D. L. and Cassimeris, L. (1999). Phosphorylation by CDK1 regulates XMAP215 function in vitro. Cell Motil. Cytoskel. 43, 310321.

Venier, P., Maggs, A. C., Carlier, M. . F, Pantaloni, D. (1994). Analysis of microtubule rigidity using hydrodynamic flow and thermal fluctuations. $J$. Biol. Chem. 269; 13353-13360.

Voter, W. A. and Erickson, H. P. (1979). Tubulin rings: curved filaments with limited flexibility and two modes of association. J. Supramol. Struct. 10, 419-431.

Voter, W. A. and Erickson, H. P. (1982). Electron microscopy of MAP2 (Microtubule-associated protein 2). J. Ultrastruct. Res. 80, 374-382.

Wang, P. J. and Huffaker, T. C. (1997). Stu2p: A microtubule-binding protein that is an essential component of the yeast spindle pole body. J. Cell Biol. 139, 1271-1280.

Waterman-Storer, C. M. and Salmon, E. D. (1997). Actomyosin-based retrograde flow of microtubules in the lamella of migrating epithelial cells influences microtubule dynamic instability and turnover and is associated with microtubule breakage and treadmilling. J. Cell Biol. 139, 417-434.

Wiese, C., Wilde, A., Moore, M. S., Adam, S. A., Merdes, A. and Zheng. Y. (2001). Role of importin- $\beta$ in coupling Ran to downstream targets in microtubule assembly. Science 291, 653-656.

Wilde, A. and Zheng, Y. (1999). Stimulatin of microtubule aster formation and spindle assembly by the small GTPase Ran. Science 284, 1359-1362.

Wille, H., Mandlekow, E.-M., Dingus, J., Vallee, R. B., Binder, L. I. and Mandelkow, E. (1992). Domain structure and antiparallel dimers of microtubule-associated protein 2 (MAP2). J. Struct. Biol. 108, 49-61. 